\title{
Derivation of Floquet Eigenvectors Displacement for Optimal Design of LC Tank Pulsed Bias Oscillators
}

\author{
Stefano Perticaroli, Nikend Luli, Fabrizio Palma \\ Department of Information Engineering, Electronics and Telecommunications, \\ Sapienza Università di Roma, Rome, Italy \\ E-mail: \{perticaroli,palma\}@die.uniromal.it \\ Received June 13, 2011; revised July 3, 2011; accepted July 22, 2011
}

\begin{abstract}
The paper presents an approximated and compact derivation of the mutual displacement of Floquet eigenvectors in a class of LC tank oscillators with time varying bias. In particular it refers to parallel tank oscillators of which the energy restoring can be modeled through a train of current pulses. Since Floquet eigenvectors are acknowledged to give a correct decomposition of noise perturbations along the stable orbit in oscillator's space state, an analytical and compact model of their displacement can provide useful criteria for designers. The goal is to show, in a simplified case, the achievement of oscillators design oriented by eigenvectors. To this aim, minimization conditions of the effect of stationary and time varying noise as well as the contribution of jitter noise introduced by driving electronics are deduced from analytical expression of eigenvectors displacement.
\end{abstract}

Keywords: Floquet Eigenvectors Noise Decomposition, Pulsed Bias Oscillator, Oscillator Phase Noise

\section{Introduction}

Noise decomposition through Floquet eigenvectors is widely acknowledged to be a correct methodology for the analytical treatment of phase noise in electronic oscillators [1]. For this reason, numerical algorithms which rely also on the Floquet eigenvectors have been developed in order to obtain accurate prediction of power density spectrum (PDS) around the fundamental oscillation frequency. A well known example is found in the commercial simulator SpectreRF that computes Floquet eigenvectors from the shooting matrix and use them as a theoretical based correction for the PNoise analysis [2]. Although electronic simulators offer results in good agreement with effective measurements, they cannot be used to infer general properties of the underlying system. Despite the great amount of work presented even recently in literature [3, 4], since the pioneeristic work of Kaertner [5] and the latter efforts accomplished by Hajimiri-Lee [6], Demir [7, 8] and Buonomo [9] not many significant theoretical contributions have really extended the capability to develop innovative techniques in the design of oscillators systems.

In authors opinion the innovation should be founded on derivation of architecture-related expressions of Floquet eigenvectors [10], leading to a proper design methodol- ogy. This approach has been limited in the past by the fact that, also in relatively simple cases, Floquet eigenvectors cannot be obtained in analytical closed form.

Mutual displacement among eigenvectors along the period of oscillation regulates indeed how noise perturbations affect the spectral purity of oscillator system. In particular projections of noise on the eigenvectors determine the PDS of the oscillator [11]. We notice that mutual displacement depends in general on the quality factor of tank and on the way the lost energy is restored, i.e. on the chosen architecture. Even if in recent years several new architectural solutions were proposed for the reduction of phase noise in CMOS technologies [12-15], none of them was justified on eigenvectors based considerations. In particular, to knowledge of the authors, in literature there is no attempt to establish a direct relationship between Floquet eigenvectors and circuit parameters for a certain class of oscillators.

In this paper we present an analytical derivation of mutual displacement of Floquet eigenvectors and of their relationship with design parameters in the class of the LC tank oscillators with pulsed bias. First, we propose to introduce a parametrical model for the pulsed bias concept. Then we extend Floquet theory for the class in study in order to analyze noise dynamics and thus to optimize 
oscillators implemented through architectures with time varying bias. Finally we validate the proposed analysis and optimization criteria through the comparison with numerical results from a dedicated Matlab simulator of introduced model.

\section{Simplified Model of LC Tank Pulsed Bias Oscillator}

In order to characterize the class of LC tank oscillators with pulsed current bias we adopt the simplified parallel RLC model in Figure 1: it presents only two state variables, corresponding to the capacitor voltage $V_{C}$ and the inductor current $I_{L}$ of tank. This assumption can be seen as a rather drastic simplification for a model of a real oscillator, nevertheless, if pulsed bias circuitry does not introduce parasitic comparable to those in tank, the additional state variables can be neglected.

In this model the energy refilling is demanded to pulsed current generator $i(t)$ which is controlled, in particular, by the capacitor voltage $V_{C}$. Crossing of a threshold $\left|V_{t h}\right|$ by the capacitor voltage, occurring at time defined as $T_{t h}$, triggers the accumulation of a fixed delay $T_{1}$. After this delay the current generator is turned on for a time $T_{2}$ at fixed amplitude $I_{\max }$. The turning on and off are assumed to be instantaneous, thus application of the ideal pulses gives rise to non derivable points of the capacitor voltage. In order to clarify the aforementioned timings and parameters of the model, a sketch of the generator current and of the resulting capacitor voltage waveform is reported in Figure 2.

The differential algebraic equations (DAE) describing dynamic of the proposed model is reported in Equation (1).

$$
\left[\begin{array}{c}
\dot{V}_{C}(t) \\
\dot{I}_{L}(t)
\end{array}\right]=\left[\begin{array}{cc}
-\frac{1}{R C} & -\frac{1}{C} \\
\frac{1}{L} & 0
\end{array}\right] \cdot\left[\begin{array}{c}
V_{C}(t) \\
I_{L}(t)
\end{array}\right]+\left[\begin{array}{c}
\frac{1}{C} \\
0
\end{array}\right] \cdot i(t)
$$

We choose to not explicitly express the large signal dependence of $i(t)$ as a function of $V_{C}$, since in this paper we are interested in a variational analysis for the study of noise only. However the existence of a stable orbit can be easily proved by means of mathematical approach for the analysis of nonlinear systems (e.g. the describing function

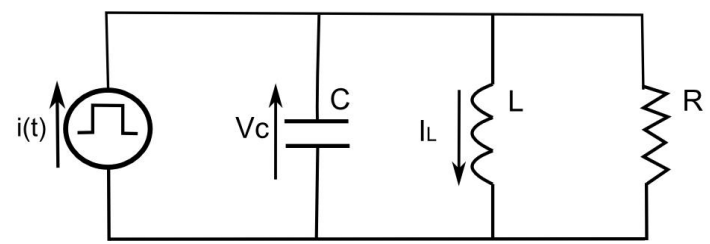

Figure 1. Model of the simplified LC tank pulsed bias oscillator.

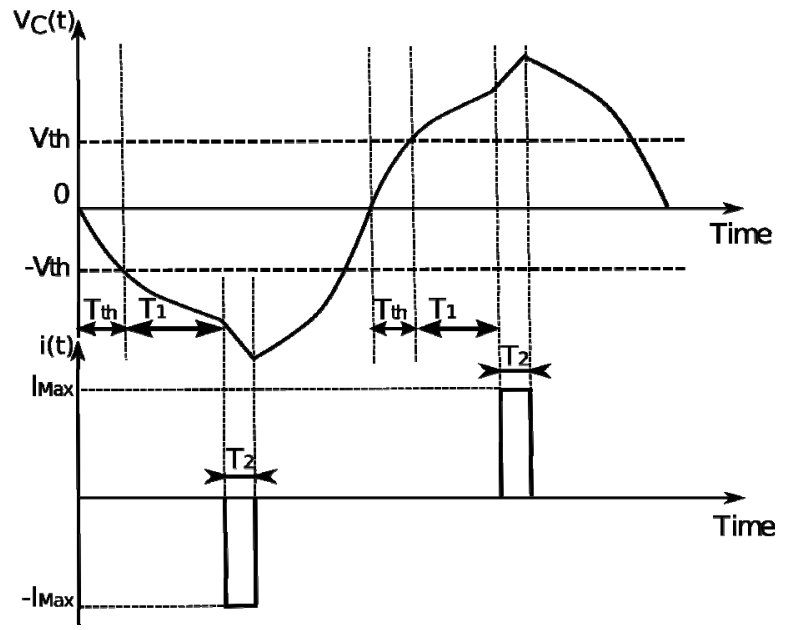

Figure 2. Sketch of capacitor voltage and current pulse of the generator in time.

technique) and will be not reported here.

In Figure 3(a) sketch of the stable orbit and the projections onto eigenvectors of superimposed noise perturbation $\underline{b}$ at certain time instant are reported. Due to the adopted symmetrical model, every half of the orbit can be subdivided into two different portion delimited by dashed lines in Figure 3. A first one corresponds to the RLC dumped evolution only and the second one corresponds to the evolution when the refilling current pulse is also active.

\section{Floquet Theory for the Study of Noise}

Floquet theory describes the periodical linear time varying (LPTV) response of oscillators systems to small perturbations superimposed on the stable oscillation orbit. Floquet eigenvectors are usually extracted from Mono-

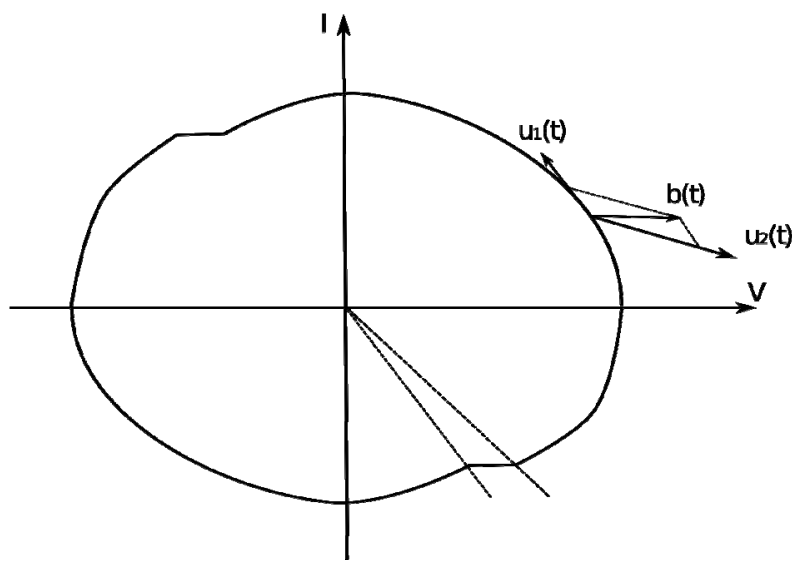

Figure 3. Sketch of the stable orbit and of the projections onto eigenvectors of superimposed noise perturbation $\underline{b}$ at certain time instant. Dashed lines indicate phase portion when pulsed bias current is active in a half of period. 
dromy matrix. In general Monodromy matrix is obtained as the product of sequence of eventually different transition matrices along one oscillation period. In our treatment Monodromy matrix is calculated by following the evolution of the state variation vector $\left[v_{C} i_{L}\right]^{T}$ in the system along the orbit, apart from non derivable points. Evolution is derived from (1) imposing $i(t)=0$ and is given by

$$
\left[\begin{array}{c}
\dot{v}_{C}(t) \\
\dot{i}_{L}(t)
\end{array}\right]=\left[\begin{array}{cc}
-\frac{1}{R C} & -\frac{1}{C} \\
\frac{1}{L} & 0
\end{array}\right] \cdot\left[\begin{array}{c}
v_{C}(t) \\
i_{L}(t)
\end{array}\right],
$$

The state transition matrix $\Phi(t)$ of dumped RLC system (2) results in

$$
\Phi(t)=\left[\begin{array}{ll}
\Phi_{11}(t) & \Phi_{12}(t) \\
\Phi_{21}(t) & \Phi_{22}(t)
\end{array}\right]
$$

Every component of (3) can be obtained by direct integration of (2) and hence assumes the form of dumped sinusoidal evolution as expressed in Equation (4)

$$
\left\{\begin{array}{l}
\Phi_{11}(t)=\frac{e^{-\mu t}}{\omega} \sqrt{\mu^{2}+\omega_{n}^{2}} \cos \left(\omega_{n} t-\varphi\right) \\
\Phi_{12}(t)=\frac{-L e^{-\mu t}}{\cos (-\varphi)} \sqrt{\mu^{2}+\omega_{n}^{2}} \sin \left(\omega_{n} t\right) \\
\Phi_{21}(t)=\frac{e^{-\mu t}}{L \omega} \sin \left(\omega_{n} t\right) \\
\Phi_{22}(t)=\frac{e^{-\mu t}}{\cos (-\varphi)} \cos \left(\omega_{n} t-\varphi\right)
\end{array}\right.
$$

where

$$
\begin{gathered}
\omega_{0}=\sqrt{\frac{1}{L C}}, \quad \omega_{n}=\frac{2 \pi}{T_{n}}=\omega_{0} \sqrt{1-\left(\frac{\mu}{\omega_{0}}\right)^{2}} \\
Q=\frac{R}{\omega_{0} L}=\frac{\omega_{0}}{2 \mu}, \quad \mu=\frac{1}{2 R C}=\frac{\omega_{0}}{2 Q} \\
\varphi=\operatorname{arctg}\left(-\frac{\mu}{\omega_{n}}\right) \approx \operatorname{arctg}\left(-\frac{\mu}{\omega_{0}}\right)=\operatorname{arctg}\left(-\frac{1}{2 Q}\right)
\end{gathered}
$$

It has to be noticed that real period $T$ differs in general from the nominal period $T_{n}$ as a main effect of the pulsed bias.

The above described model holds until current pulse edges are reached. Since at this time instants the capacitor voltage is not derivable, we will need to introduce Interface matrices $[16,17]$ to properly describe state evolution.

In the simplified adopted model only two Floquet eigenvectors exist, with two corresponding eigenvalues.
We call "first eigenvector", $\underline{u}_{1}(t)$, the one which is tangent to the orbit with unitary eigenvalue and the "second eigenvector", $\underline{u}_{2}(t)$, the one with corresponding eigenvalue lower than 1 . Once the evolution of the eigenvectors is available, we may project the perturbation $\underline{b}(t)$ multiplying it by inverse eigenvectors. The effect of noise projection is a state deviation which evolves as the eigenvector itself and sum in square to further noise projections along the orbit. Projections depend on the instant when $\underline{b}(t)$ is applied, so that the system appears as periodic time variant. In Figure 4(a) sketch of power density spectrum of the eigenvectors is reported [11]. Contribution to the PDS of noise projected on the first eigenvector, can be described by a transfer function with square modulus $1 / \omega^{2}$, where is the offset with respect to the fundamental. We notice that at very low offset a cut-off must be assumed, not reported in the figure, due to nonlinear behaviour of the system for large values of phase deviation [7]. Noise projected on the second eigenvector can be instead described by a transfer function with square modulus $1 /\left(\gamma_{2}^{2}+\omega^{2}\right)$, where $\gamma_{2}$ is the pole pulsation related to the second Floquet multiplier.

As a result the main contribution to the overall power density spectrum at frequencies close to the fundamental arises from projection on the first eigenvector. On the contrary contribution arising from the second eigenvector becomes relevant only at high frequency offsets $\left(\omega>>\gamma_{2}\right)$ due to its low-pass shape with respect to the fundamental.

Only noise contributions parallel to $\underline{u}_{2}$ produce null contributions on $\underline{u}_{1}$ and thus do not increase the phase noise [1]. Floquet decomposition points out that eigenvectors, in general, are not orthogonal. As a result, assuming a white noise current generator in parallel to the RLC resonator, as it will be discussed in next section, a noise contribution in the direction $\left[\begin{array}{ll}1 & 0\end{array}\right]^{T}$ (the voltage

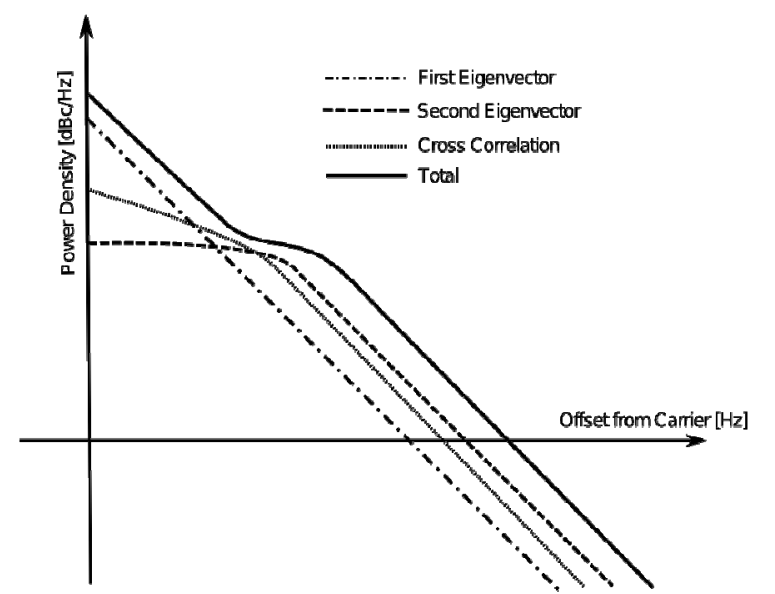

Figure 4. Sketch of power density spectrum contributions due to the eigenvectors. 
variation axis) applied in correspondence of the voltage maximum may not yield to a null projection on $\underline{u}_{1}$.

These considerations lead to investigate a pulsed bias strategy, designed to activate the bias only when the projection of noise related to the biasing electronics on the first eigenvector is at least around a minimum. Nevertheless a complete evaluation of the proposed architectcture must take into account also the effect of eigenvectors displacement due to the pulsed bias on all the other noise sources present in the circuit.

As formerly stated, the eigenvectors evolutions are almost everywhere derived from state transition matrix of the dumped RLC system except for time instants when the generator switches on/off. Since an oscillator has no time reference, in the adopted model we may assume that at $t=0$ the first eigenvector must be equal to $\underline{u}_{1}(0)=$ $\left[V_{u 1}(0) I_{u 1}(0)\right]=k\left[\begin{array}{ll}10\end{array}\right]^{T}, k \in \mathbb{R}$ with null current component (an eigenvector can always be defined by means of a proportionality constant). Until the first discontinuity, occurring at $t=T_{t h}+T_{1}$, eigenvectors evolutions can then be described by the following equations normalized to amplitude of superimposed perturbation

$$
\begin{aligned}
& \underline{u}_{1}(t)=\left[\begin{array}{l}
V_{u l}(t) \\
I_{u l}(t)
\end{array}\right]=\left[\begin{array}{c}
e^{-\mu t} \cos \left(\omega_{n} t-\varphi\right) \\
\frac{e^{-\mu t}}{F} \sin \left(\omega_{n} t\right)
\end{array}\right] \\
& \underline{u}_{2}(t)=\left[\begin{array}{l}
V_{u 2}(t) \\
I_{u 2}(t)
\end{array}\right]=\left[\begin{array}{c}
e^{-\mu t} \cos \left(\omega_{n} t-\varphi+\beta\right) \\
\frac{e^{-\mu t}}{F} \sin \left(\omega_{n} t+\beta\right)
\end{array}\right]
\end{aligned}
$$

where coefficient $F$ depends on the quality of the tank and on resonating elements as in (7) and $\beta$ represents the phase displacement between eigenvectors.

$$
F=\sqrt{\frac{L}{C}} \cdot \sqrt{1+\frac{1}{(2 Q)^{2}}}
$$

\section{Phase Displacement between Eigenvectors as a Function of Pulse Paramaters}

Figures 5 and $\mathbf{6}$ show a sketch of the evolutions respectively of first and second eigenvector. The effect of turning on and off of the bias current, taking place at $t=T_{\text {th }}$ $+T_{1}$ and $t=T_{t h}+T_{1}+T_{2}$ respectively, corresponds to discontinuities of eigenvectors states, in direction $\left[\begin{array}{ll}1 & 0\end{array}\right]^{T}$ (the $V$ voltage variation axis). Due to the introduction of the Interface matrix theory [16], and as shown in Figure 7 , we have to consider that any state variation along each eigenvector, evolved from $t=0$ until $T_{t h}$, produces a delay $t$ with respect to the crossing of the threshold $\left|V_{t h}\right|$.

The same delay is reported at the time of the turning on and off of the current generator. The delay is calculated as

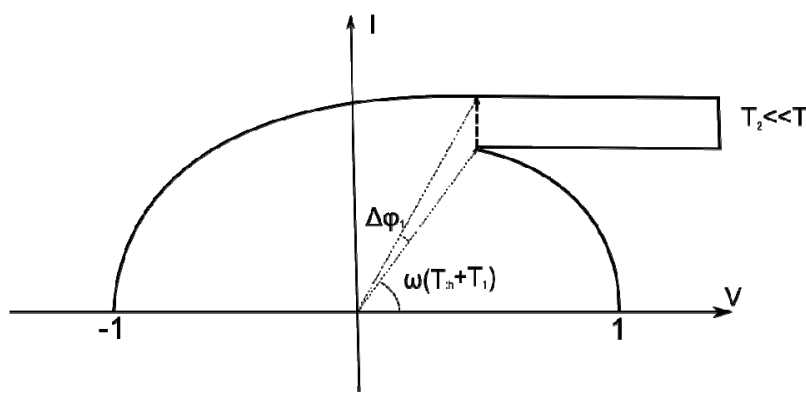

Figures 5. Sketch of evolution of the first eigenvector for a half of the period.

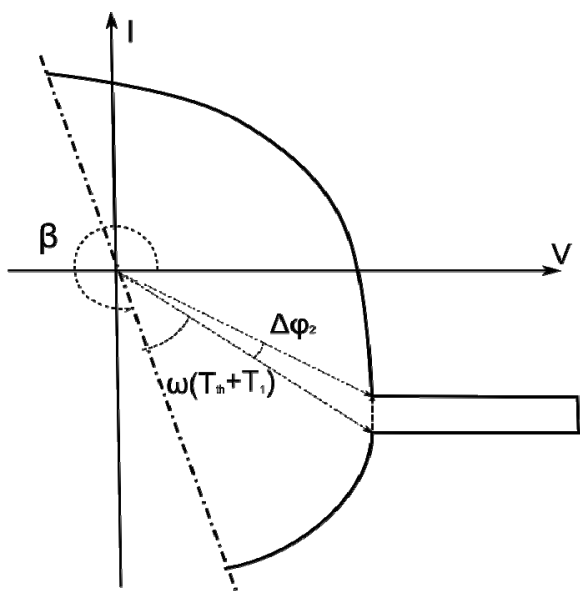

Figures 6. Sketch of evolution of the second eigenvector for a half of the period.
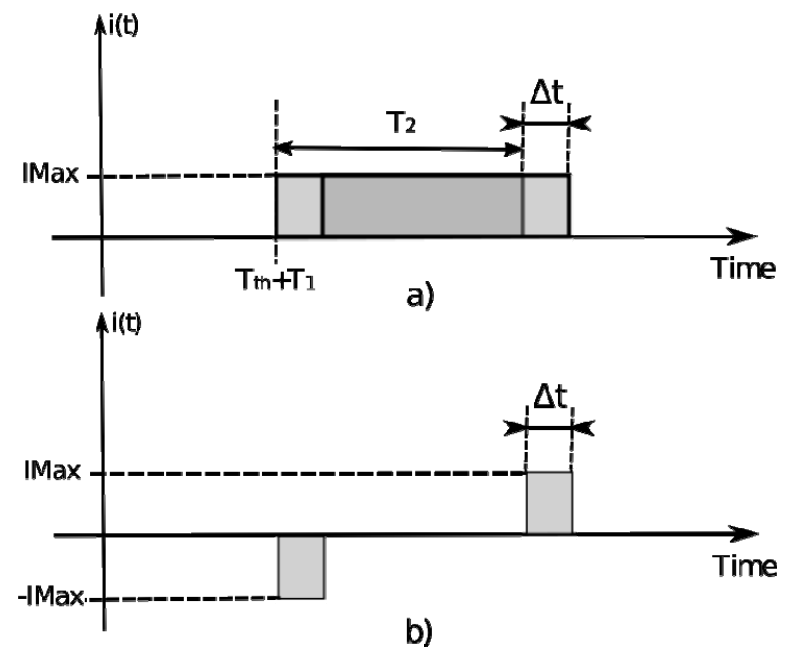

Figure 7. Current of the generator in time with delay due to the variation (a) and additional contributions at the pulse edges (b).

the ratio of voltage variation to the time derivative of the capacitor voltage at threshold crossing. Using a smart notation with subscript $J=1,2$ where $X_{J=1}=0$ and $X_{J=2}=$ $\beta$, delay for both eigenvectors results in 


$$
\Delta t_{J}=\frac{e^{-\mu T_{t h}}}{\left.\dot{V}_{C}\right|_{t=T_{t h}}} \cos \left(\omega_{n} T_{t h}-\varphi+X_{J}\right)
$$

Implicit voltage variation at numerator ensures dimensional balance in Equation (8). As a result of the delay, at the pulse edges two additional contributions opposite in sign sum to the former state variation. Since we are dealing with a pulsed bias for hypothesis, we now assume $T_{2}<<T$ and then we neglect the relative phase rotation between the pulse edges in both eigenvectors evolutions. As depicted in Figure 5, this allows us to approximate the effect of the bias current pulse as a unique discontinuity of the state in direction $\left[\begin{array}{ll}0 & 1\end{array}\right]^{T}$ (the I current variation axis). We hence calculate discontinuity amplitude for both eigenvectors using (6) and (8) as the sum of opposite contributions approximating sinusoidal function for small $T_{2}$ in

$$
\Delta I_{J}=\frac{\mathrm{I}_{\max }}{\left.C \dot{V}_{C}\right|_{t=T_{t h}} F} e^{-\mu T_{t h}} \omega_{n} T_{2} \cos \left(\omega_{n} T_{t h}-\varphi+X_{J}\right)
$$

The overall state variation, including the effect due to discontinuities, evolves along a new orbit. The second part of the evolution can be obtained for both the eigenvectors as

$$
\underline{u}_{J}(t)=\left[\begin{array}{c}
V_{J}(t) \\
I_{J}(t)
\end{array}\right]=\left[\begin{array}{c}
\mathrm{A}^{-\mu t} \cos \left(\omega_{n} t-\varphi+X_{J}+\Delta \varphi_{J}\right) \\
\frac{\mathrm{A}}{F} e^{-\mu t} \sin \left(\omega_{n} t+X_{J}+\Delta \varphi_{J}\right)
\end{array}\right]
$$

where $\mathrm{A}$ and $\Delta \varphi_{J}$ are unknown general solution parameters.

We notice that at $t=T_{t h}+T_{1}$ the voltage components remain unaltered due to the assumption $T_{2}<<T$, whereas the current components of state variations are affected by the $\Delta I_{J}$ drops. This observation leads to define a system of two non-linear equations in the two unknown $\mathrm{A}$ and $\Delta \varphi_{J}$ :

$$
\left\{\begin{array}{l}
\mathrm{A} e^{-\mu\left(T_{t h}+T_{1}\right)} \cos \left(\omega_{n} T_{t h}+\omega_{n} T_{1}-\varphi+X_{J}+\Delta \varphi_{J}\right) \\
=e^{-\mu\left(T_{t h}+T_{1}\right)} \cos \left(\omega_{n} T_{t h}+\omega_{n} T_{1}-\varphi+X_{J}\right) \\
\frac{\mathrm{A}}{F} e^{-\mu\left(T_{t h}+T_{1}\right)} \sin \left(\omega_{n} T_{t h}+\omega_{n} T_{1}+X_{J}+\Delta \varphi_{J}\right) \\
=\frac{1}{F} e^{-\mu\left(T_{t h}+T_{1}\right)} \sin \left(\omega_{n} T_{t h}+\omega_{n} T_{1}+X_{J}\right)+\Delta I_{J} .
\end{array}\right.
$$

In order to achieve an approximate solution of system (11) we expand the trigonometric function containing $\Delta \varphi_{J}$ in Mac Laurin series, limited to the first order and obtain:

$$
\Delta \varphi_{J}=\frac{\Delta I_{J} F \cos \left(\omega_{n} T_{t h}+\omega_{n} T_{1}-\varphi+X_{J}\right)}{\cos (-\varphi)+\Delta I_{J} F \sin \left(\omega_{n} T_{t h}+\omega_{n} T_{1}-\varphi+X_{J}\right)} .
$$

Since in most of practical cases tank quality factor $Q$ is at least greater than 5 we further observe that

$$
\begin{aligned}
& \cos (-\varphi) \approx \cos \left(\operatorname{arctg}\left(\frac{1}{2 Q}\right)\right) \approx \cos \left(\frac{1}{2 Q}\right) \approx 1 \\
& \Delta I_{J} F<<1
\end{aligned}
$$

thus we may reformulate (12) neglecting terms due to pulse in the sum at denominator to solve the general parameter $\Delta \varphi_{J}$ for both the eigenvector as

$$
\begin{aligned}
\Delta \varphi_{1} & \cong \frac{\Delta I_{1} F \cos \left(\omega_{n} T_{t h}+\omega_{n} T_{1}-\varphi\right)}{\cos (-\varphi)} \\
\Delta \varphi_{2} & \cong \frac{\Delta I_{2} F \cos \left(\omega_{n} T_{t h}+\omega_{n} T_{1}-\varphi+\beta\right)}{\cos (-\varphi)}
\end{aligned}
$$

where $\Delta \varphi_{1}$ and $\Delta \varphi_{2}$ account the phase discontinuities due to the bias current pulse, respectively, for the first and the second eigenvector.

Since, by the definition, eigenvectors are periodic, and, due to the symmetry of the adopted model in the two halves of period they cover the same phase angle, we may infer that phase discontinuities caused by the bias current pulse must be equal, i.e.

$$
\Delta \varphi_{1}=\Delta \varphi_{2}
$$

From these observations, substituting the condition (15) in Equation (14) and expanding $\Delta I_{J}$ drops, it is straightforward to obtain the value of the phase displacement between the eigenvectors $\underline{u}_{1}$ and $\underline{u}_{2}$, at least out of the orbit portion when pulsed current is active as

$$
\beta=-\left(2 \omega_{n} T_{t h}+\omega_{n} T_{1}-2 \varphi\right) \text {. }
$$

We want to remark that in Equation (16) $\beta$ is only a function of the threshold crossing time, of the delay of current pulse application, and of the tank quality factor (related to parameter $\varphi$ ).

\section{Optimal Phase Displacement between Eigenvectors}

Phase displacement between eigenvectors becomes here the main tool toward the paper goal to offer design insights for the reduction of phase noise which, in our treatment, means to minimize projection of noise on the first eigenvector. To this aim we are now going to demonstrate optimum conditions on phase displacement between eigenvectors, in order to reduce noise due to bias, parasitic resistance and jitter on accumulation of delay time $T_{1}$.

We assume perturbations to be realizations of white Gaussian noise processes with zero time average. This is not a restrictive assumption. In fact, also noise sources 
with non-delta autocorrelations, i.e. $1 / f^{\alpha}$ with $\alpha \in \mathbb{R}^{+}$, may be taken into account through the projections on the eigenvectors. Referring to [5], in particular Sections 6 and 7 , the power density of a flicker noise source is obtained as an infinite sum of autocorrelation spectra of statistically independent Ornstein-Uhlenbeck processes (that are also Gaussian processes). Since we are dealing with a parallel RLC tank, perturbations are introduced as parallel noise current sources. Such noise current sources cause a variation of the capacitor voltage, hence we model the normalized noise perturbation through a constant vector

$$
\underline{b}=\left[\begin{array}{c}
V_{b} \\
I_{b}
\end{array}\right]=\left[\begin{array}{l}
1 \\
0
\end{array}\right] .
$$

In order to determine the PDS we need to calculate the projection of vector $\underline{b}$ on the base formed by the two eigenvectors. Integration along the orbit of the square value of the projection on $\underline{u}_{1}$ leads to the $c_{1}$ coefficient of Demir [7], while projection on $\underline{u}_{2}$ must be multiplied before integration by a properly evaluated exponential factor [11].

We first search for the condition which zeroes the projection on $\underline{u}_{1}$ of noise due to the bias current, usually the largest source of noise in integrated technologies. Bias current noise is cyclostationary because it arises only during the bias pulse. Assuming again $T_{2}<<T$ we may consider constant the projection angle during the pulse, then integration reduces to the multiplication of the projection by time $T_{2}$. We notice that noise projection has to be performed after the turning on of the bias current, after the eigenvector discontinuity in $T_{t h}+T_{1}$ has occurred. As depicted in Figures 5 and $\mathbf{6}$, the additional component is in the direction $\left[\begin{array}{ll}1 & 0\end{array}\right]^{T}$, thus the necessary and sufficient condition which ensures that once added the Interface matrix component the eigenvector has null current component is

$$
\underline{u_{2}}\left(T_{t h}+T_{1}\right) \cdot \underline{b}=0 \rightarrow \underline{u_{2}}\left(T_{t h}+T_{1}\right) \cdot\left[\begin{array}{l}
1 \\
0
\end{array}\right]=0 .
$$

Following the definition of $\underline{u}_{2}$ in Equation (6) and substituting in its expression the phase displacement of Equation (16), condition (18) is equivalent to:

$$
\begin{gathered}
\omega_{n}\left(T_{t h}+T_{1}\right)+\beta \\
=\omega_{n}\left(T_{t h}+T_{1}\right)-\left(2 \omega_{n} T_{t h}+\omega_{n} T_{1}-2 \varphi\right)=0 \\
\downarrow \\
\omega_{n} T_{t h}=2 \varphi .
\end{gathered}
$$

Equation (19) states that, in possible implementations of pulsed bias oscillators, the $\left|V_{t h}\right|$ threshold should be chosen around the zero crossing of the oscillation voltage.

We may further search for the minimization of the contribution arising from parasitic resistance, which re- mains active all along the orbit. We recall that, from Equation (16) and following condition $T_{2}<<T$ which led to (15) phase displacement between eigenvectors remains constant along the entire evolution except for region where the pulsed current bias is active.

However, since we assumed bias active for a short time compared to period, the projections of noise on the two eigenvectors, respectively $u_{1 p}$ and $u_{2 p}$, are given by

$$
\left\{\begin{array}{l}
u_{1 p}(t)=\frac{\sin \left(\beta+\omega_{n} t\right)}{\sin (\beta)} \\
u_{2 p}(t)=\frac{\sin \left(\omega_{n} t\right)}{\sin (\beta)}
\end{array}\right.
$$

then we obtain the integral of the square of the projections

$$
\left\{\begin{array}{l}
\int_{0}^{T} u_{1 p}(t)^{2} \mathrm{~d} t=\frac{1}{\sin ^{2}(\beta)} \int_{0}^{T} \sin ^{2}\left(\beta+\omega_{n} t\right) \mathrm{d} t=\frac{1}{\sin ^{2}(\beta)} \frac{T}{2} \\
\int_{0}^{T} u_{2 p}(t)^{2} \mathrm{~d} t=\frac{1}{\sin ^{2}(\beta)} \int_{0}^{T} \sin ^{2}\left(\omega_{n} t\right) \mathrm{d} t=\frac{1}{\sin ^{2}(\beta)} \frac{T}{2}
\end{array}\right.
$$

As expected the two terms in Equation (21) are equal. The first one is the Demir $c_{1}$ coefficient [7], while the second is only a majorant term in the expression of phase noise due to $\underline{u}_{2}$.

The actual period $T$ depends on the angle $\beta$ between the eigenvectors, however in hypothesis $Q>5$ it results very close to $T_{n}$ (the nominal period). This leads to state that factor $\sin ^{2}(\beta)$ predominates the result of integration and allows us to infer that minimization of phase noise distribution due to a stationary noise source occurs if the condition $\beta= \pm \pi / 2$ holds.

Further considerations can be derived from this result and from condition expressed in (19). For example, we notice that a jitter noise contribution arises from the electronic circuit when we introduce the delay $T_{1}$. This jitter is proportional to the delay itself and inversely proportional to the power used to accumulate the delay [18]. The choice to avoid any delay may be of interest in order to save an additional source of noise and to reduce energy dissipation [19]. Imposing $T_{1}=0$ in combination with condition (21) we obtain that the optimum choice is to set

$$
\left\{\begin{array}{l}
T_{1}=0 \\
\beta=-2\left(\omega_{n} T_{t h}-\varphi\right)=-\frac{\pi}{2} \Rightarrow \omega_{n} T_{t h}-\varphi=\frac{\pi}{4}
\end{array}\right.
$$

It results that condition on minimization of stationary noise (21) together with condition on minimization of accumulated jitter can be simultaneously satisfied only for a very low quality factor $(Q=0.5)$. Then in any prac- 
tical case a suboptimal condition must be searched, e.g. accepting a non zero delay time $T_{1}$.

Hence the last optimum condition we can search for is derived from minimization of both resistance noise and bias current noise for a given quality factor $Q$ and in presence of a delay time of the current pulse $T_{1}$. Using results from Equations (19) and (21) in Equation (16) we obtain

$$
\omega_{n} T_{1}=\frac{\pi}{2}+2 \varphi=\frac{\pi}{2}-2 \operatorname{arctg}\left(\frac{1}{2 Q}\right)
$$

\section{Numerical Comparison and Discussion}

In order to evaluate the accuracy of the proposed analytical expressions we need to compare them with numerical simulations. In fact, at the knowledge of the authors, there is no measurement setup and/or post-processing that can extract eigenvectors from measure of physical quantities in a real circuital implementation.

Moreover there are no commercial circuit simulator which can compute Floquet eigenvectors in presence of discontinuities in space state. Then we developed a dedicated Matlab simulator for the model defined in Section III. The simulator derives the Monodromy matrix through a shooting algorithm which use Interface matrices for the treatment of discontinuities.

Even if the proposed analysis is not dependent on the oscillation period, in the following simulations we fixed frequency at $f_{n}=\omega_{n} /(2 \pi)=5 \mathrm{GHz}$. Along with frequency, we choose to keep constant quality factor $Q=7.5$, pulse duration $T_{2}=T / 15$ and amplitude $I_{\max }=60 \mathrm{~mA}$ ensuring the oscillator to be refilled by a fixed amount of energy and to be perturbed by the same amount of noise power. Moreover in case $V_{\text {th }} \neq 0 \mathrm{~V}$ we keep constant also the sum $T_{t h}+T_{1}$, allowing to reach the same limit cycle of $V_{t h}=0$ $\mathrm{V}$ simulations set. The two case of study, respectively $V_{t h}$ $\neq 0 \mathrm{~V}$ and $V_{\text {th }} \neq 0 \mathrm{~V}$, differ only in the starting point with respect to period of $T_{1}$ delay accumulation and in the weight of jitter contribution which is proportional to $T_{1}$. All kind of noise sources are modeled as parallel current generators that adds to the model in Figure 1. Stationary noise is introduced as thermal noise of the parasitic resistance depending on quality factor $Q$ through $4 k_{B} T_{a m b} / R$ [A $\left.\mathrm{A}^{2} / \mathrm{Hz}\right]$ relation $\left(k_{B}\right.$ is Boltzmann constant $T_{a m b}$ is ambient temperature in Kelvin) whereas the cyclostationary noise is introduced as shot noise (modeling eventual devices) related to current $I_{\max }$ through $2 q I_{\max }\left[\mathrm{A}^{2} / \mathrm{Hz}\right]$ relation ( $q$ is electron charge). Jitter noise source is instead considered as an additional time shift due to the accumulation of both $T_{1}$ and $T_{2}$ delay and it is added to the one due to evolution of the initial variation until time of threshold crossing $T_{t h}(8)$. Jitter source is the result of the charging of a capacitor through a dissipative media (again assume a MOS device) so it has been modeled through $8 k_{B} T_{a m b} \gamma T_{1}\left(K_{M O S} / I_{D}^{3}\right)^{0.5}[\mathrm{~s}]$ relation where is $\gamma$ transistor noise constant, $K_{M O S}$ and $I_{D}$ are respectively the large signal current gain and drain current of MOS device. We choose to fix drain current to $I_{D}=I_{\max } / 10=6 \mathrm{~mA}$. In fact the eventual auxiliary circuit suited for the introduction of desired delay must necessarily have a lower power consumption with respect the refilling process of the tank in a real design.

In Figure 8 the phase displacement $\beta$ is reported in function of $T_{1}$ normalized to period $T$ in two cases $V_{t h}=0$ $\mathrm{V}$ and $V_{t h}=0.75 \mathrm{~V}$.

Amplitude and period of oscillation, as stated before, vary in function of pulse position between $[2.45 \mathrm{~V}, 3.11$ $\mathrm{V}$ ] and [4.8 GHz, 5.2 GHz] respectively.

It can be observed a good match between simulated and calculated (16) trend with a maximum absolute error of about 13 in the evaluated range for case $V_{t h}=0.75 \mathrm{~V}$.

Former considerations on factor $\sin ^{2}(\beta)$ in case $V_{t h}=0$ $\mathrm{V}$ suggest that in correspondence of $T_{1} / T \approx 0.223$ when $\beta \approx \pi / 2$ a minimization of noise projection on the first eigenvector should occur. Moreover for $T_{1} / T \approx 0.223$ the maximum of amplitude and the nominal frequency are obtained. In case $V_{t h}=0.75 \mathrm{~V}$ the enhancement of time shift (8) due to late starting point $T_{t h}$ of delay accumulation overwhelm any reduction of jitter weight due to shorter $T_{1}$.

In Figure 9 the simulated PDS of the oscillator is reported for three typical frequency offsets $(100 \mathrm{KHz}$, $1 \mathrm{MHz}$ and $10 \mathrm{MHz}$ ) from the fundamental in function of

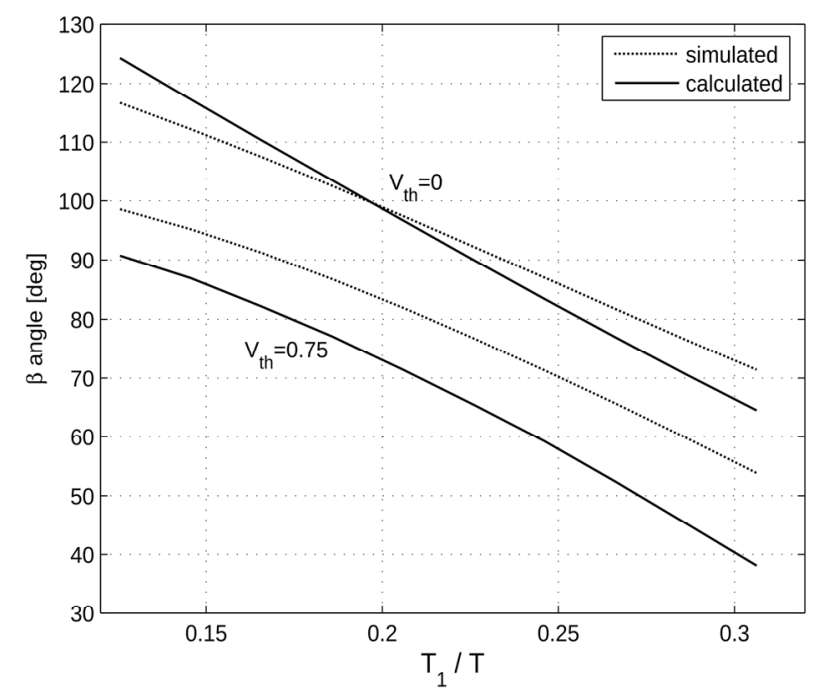

Figure 8. Eigenvectors phase displacement out of the fast region simulated (dotted trace) and calculated (continue trace) in function of normalized pulse position $T_{1}$ for $T_{2}=$ $T / 15, Q=7.5$ in cases $V_{t h}=0 \mathrm{~V}$ and $\left|V_{t h}\right|=0.75 \mathrm{~V}$. 


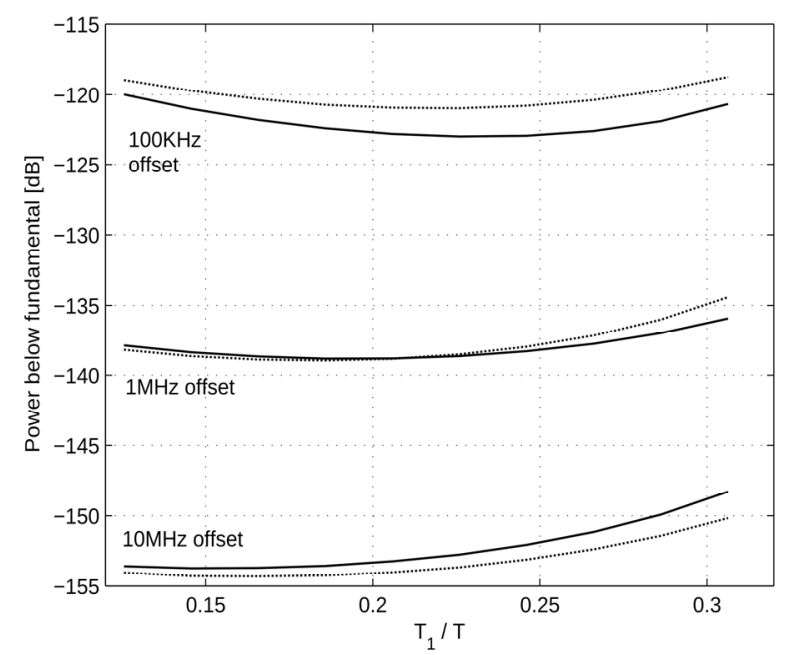

Figure 9. Simulated PDS evaluated at three different offset from fundamental frequency in function of normalized pulse position $T_{1}$ for $T_{2}=T / 15, Q=7.5$ in cases $V_{t h}=0 \mathrm{~V}$ (continue trace) and $\left|V_{t h}\right|=0.75 \mathrm{~V}$ (dotted trace).

$T_{1}$ in case of $V_{t h}=0 \mathrm{~V}$ and $V_{t h}=0.75 \mathrm{~V}$.

PDS has been computed following [11]. It can be observed that the continue trace related to the lowest offset, where the major contribution to PDS arises from projection on first eigenvector, exhibits the minimum exactly in correspondence of the prediction made by means of $\beta \approx$ $\pi / 2$ criterion for $V_{t h}=0 \mathrm{~V}$. At higher offsets contributions arising from projections on cross-correlations between eigenvectors and on second eigenvector become preponderant and the minima shift toward earlier $T_{1} / T$ ratio for both $V_{t h}$ cases. However it can be noticed that the $V_{t h}=0$ $\mathrm{V}$ case achieves the better performance at the lowest offset whereas the $V_{t h}=0.75 \mathrm{~V}$ case reaches the best results at large offsets for quite all $T_{1} / T$ ratio.

In order to justify this observed trend we propose to monitor noise introduced through first eigenvector (referring to $u_{1 p}(t)$ noise projection) in term of injected level of energy from all noise sources on first eigenvector.

In Figure 10 the energy injected along the normalized period on first eigenvector by perturbation vector $\underline{b}$ is reported in cases $V_{t h}=0 \mathrm{~V}$ and $V_{t h}=0.75 \mathrm{~V}$ for $T_{1} / T=$ $0.24, T_{2}=T / 15, Q=7.5$. It can be noticed that the zero of injected energy occurs during the current bias pulse only in case $V_{t h}=0 \mathrm{~V}$. For any $V_{t h} \neq 0 \mathrm{~V}$ the zero shifts toward later time instant. Moreover, as a result of non orthogonal phase displacement between eigenvectors in case $V_{t h} \neq 0 \mathrm{~V}$ the maxima of injected energy on first eigenvector (when only stationary and jitter noise sources are present) can be greatly increased, thus degrading phase noise especially at low offsets. This observation is congruent with former defined design criterion (19) and validates the proposed analysis.

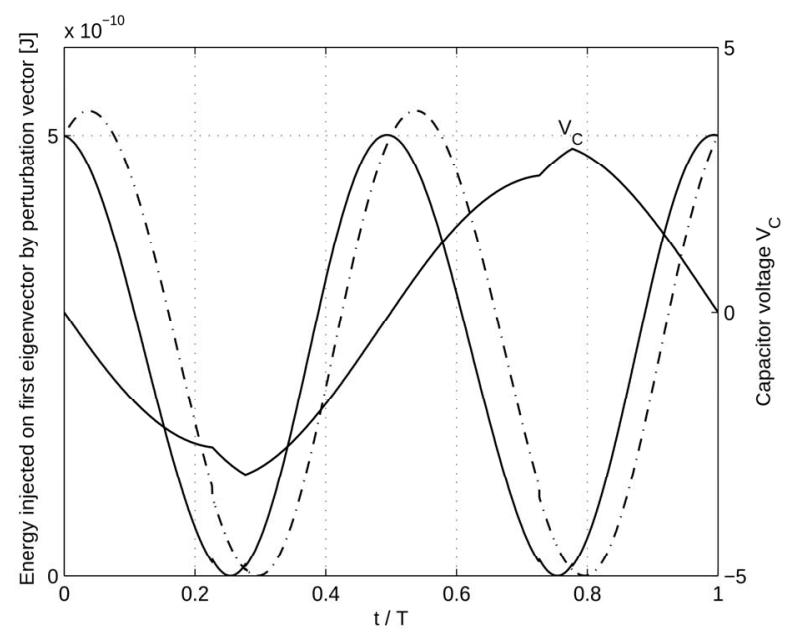

Figure 10. Simulated energy injected on first eigenvector by perturbation vector $\underline{b}$ in cases $V_{t h}=0 \mathrm{~V}$ (continue trace) and $\left|V_{t h}\right|=0.75 \mathrm{~V}$ (dotted trace) on left y-axis and capacitor voltage $V_{C}$ right $y$-axis along oscillation period for $T_{1} / T=0.24$, $T_{2}=T / 15, Q=7.5$.

\section{Concluding Remarks}

The paper presented an approximated and compact derivation of the mutual displacement of Floquet eigenvectors in the class of parallel RLC tank oscillators with pulsed current bias. Mutual displacement was proved to be strongly connected with the chosen architecture and consequently to determine phase noise distribution. As appreciable result, the derived expression of displacement is compact and straightforward, thus it can suggest primary guide lines for designers in the field of oscillators architectures with time varying bias.

In particular we demonstrated conditions for minimization of stationary as well as cyclostationary noise. Also the jitter noise introduced in the positioning of the pulsed bias is taken into account and its relation with noise projections on the eigenvectors is determined. Minimization conditions were formulated using parameters of the proposed model for the pulsed bias class, allowing to directly infer the circuit design. Finally the analytical results are compared with a dedicated simulator, showing that proposed criteria for noise reduction are congruent with observed trend in the simulated PDS.

Future works will provide developments and extensions of the proposed analytical noise treatment to VCOs and PLLs systems.

\section{References}

[1] G. J. Coram, "A Simple 2-D Oscillator to Determine the Correct Decomposition of Perturbations into Amplitude and Phase Noise," IEEE Transactions on Circuits and 
Systems-I: Fundamental Theory and Applications, Vol. 48, No. 7, July 2001, pp. 896-898.

doi:10.1109/81.933331

[2] Affirma ${ }^{\mathrm{TM}}$ RF Simulator (SpectreRFTM) User Guide, Product Version 4.4.6, June 2000, pp. 696-699.

[3] F. O'Doherty and J. P. Gleeson, "Phase Diffusion Coefficient for Oscillators Perturbed by Colored Noise," IEEE Transactions on Circuits and Systems-II: Express Briefs, Vol. 54, No. 5, May 2007, pp. 435-439.

[4] T. Djurhuus, V. Krozer, J. Vidkjaer and T. K. Johansen, "Oscillator Phase Noise: A Geometrical Approach," IEEE Transactions on Circuits and Systems-I: Regular Papers, Vol. 56, No. 7, July 2009, pp. 1373-1382. doi:10.1109/TCSI.2008.2006211

[5] F. X. Kaertner, "Analysis of White And $f-\alpha$ Noise in Oscillators," International Journal of Circuit and Theory Applications, Vol. 18, No. 5, March 1990, pp. 485-519. doi: $10.1002 /$ cta.4490180505

[6] T. H. Lee and A. Hajimiri, "Oscillator Phase Noise: A Tutorial," IEEE Journal of Solid-State Circuits, Vol. 35, No. 6, March 2000, pp. 326-336. doi:10.1109/4.826814

[7] A. Demir, "Floquet Theory and Non-Linear Perturbation Analysis Foroscillators with Differential-Algebraic Equations," International Journal of Circuit and Theory Applications, Vol. 28, No. 2, March 2000, pp. 163-185. doi:10.1002/(SICI)1097-007X(200003/04)28:2<163::AI D-CTA101>3.0.CO;2-K

[8] A. Demir, A. Mehrotra and J. S. Roychowdhury, "Phase Noise in Oscillators: A Unifying Theory and Numerical Methods for Characterization," IEEE Transactions on Circuits and Systems-I: Fundamental Theory and Applications, Vol. 47, No. 5, May 2000, pp. 655-674. doi: $10.1109 / 81.847872$

[9] A. Buonomo, "Nonlinear Analysis of Voltage-Controlled Oscillators: A Systematic Approach," IEEE Transaction on Circuit and Systems-I: Regular Papers, Vol. 55, No. 6, June 2008, pp. 1659-1670.

[10] A. Carbone, A. Brambilla and F. Palma, "Using Floquet Eigenvectors in the Design of Electronic Oscillators," 2005 IEEE 7th CAS Symposium on Emerging Technologies: Circuits and Systems for $4 G$ Mobile Wireless
Communications, St. Petersburg, 23-24 June 2005, pp. 100-103.

[11] A. Carbone and F. Palma, "Considering Orbital Deviations on the Evaluation of Power Density Spectrum of Oscillators," IEEE Transactions on Circuits and SystemsII: Express Briefs, Vol. 53, No. 6, June 2006, pp. 438442.

[12] B. Razavi, "A Study of Phase Noise in CMOS Oscillators," IEEE Journal of Solid-State Circuits, Vol. 31, No. 3, March 1996, pp. 331-343. doi:10.1109/4.494195

[13] R. Aparicio and A. Hajimiri, "A Noise-Shifting Differential Colpitts VCO," IEEE Journal of Solid-State Circuits, Vol. 37, No. 12, December 2002, pp. 1728-1736. doi:10.1109/JSSC.2002.804354

[14] S. K. Magierowski and S. Zukotynski, "CMOS LC-Oscillator Phase Noise Analysis Using Nonlinear Models," IEEE Transactions on Circuits and Systems-I: Regular Papers, Vol. 51, No. 4, April 2004, pp. 664-677. doi:10.1109/TCSI.2004.826209

[15] T. H. Lee, "Design of CMOS Radio-Frequency Integrated Circuits," Cambridge Press, Cambridge, 1998, pp. 669677.

[16] A. Carbone and F. Palma, "Discontinuity Correction in Piecewise-Linear Models of Oscillators for Phase Noise Characterization," International Journal of Circuit Theory and Applications, Vol. 35, No. 1, 2007, pp. 93-104. doi:10.1002/cta.383

[17] R. I. Leine, D. H. Van Campen and B. L. Van der Vrande, "Bifurcations in Nonlinear Discontinuous Systems," Nonlinear Dynamics, Vol. 23, No. 2, October 2000, pp. 105164. doi:10.1023/A:1008384928636

[18] M. Aleksic, N. Nedovic K. W. Current and V. G. Oklobdzija, "Jitter Analysis of Nonautonomous MOS CurrentMode Logic Circuits," IEEE Transaction on Circuit and Systems-I: Regular Papers, Vol. 55, No. 10, November 2008, pp. 3038-3049.

[19] S. Perticaroli and F. Palma, "Phase and Quadrature Pulsed Bias LC-CMOS VCO," SCIRP Circuit and Systems, Vol. 2, No. 1, January 2011, pp. 18-24. doi:10.4236/cs.2011.21004 